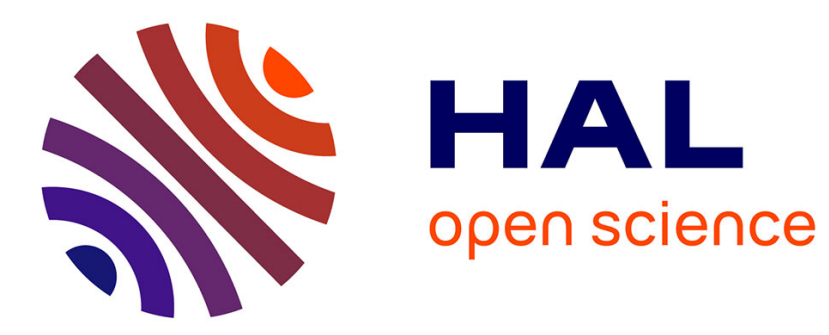

\title{
Localized interface modes in one-dimensional hyperuniform acoustic materials
}

S Kuznetsova, Jean-Philippe Groby, L García-Raffi, Vicente Romero-García

\section{To cite this version:}

S Kuznetsova, Jean-Philippe Groby, L García-Raffi, Vicente Romero-García. Localized interface modes in one-dimensional hyperuniform acoustic materials. Journal of Physics D: Applied Physics, 2021, 54 (31), pp.315303. 10.1088/1361-6463/ac006d . hal-03436155

\section{HAL Id: hal-03436155 \\ https://hal.science/hal-03436155}

Submitted on 22 Nov 2021

HAL is a multi-disciplinary open access archive for the deposit and dissemination of scientific research documents, whether they are published or not. The documents may come from teaching and research institutions in France or abroad, or from public or private research centers.
L'archive ouverte pluridisciplinaire $\mathbf{H A L}$, est destinée au dépôt et à la diffusion de documents scientifiques de niveau recherche, publiés ou non, émanant des établissements d'enseignement et de recherche français ou étrangers, des laboratoires publics ou privés. 


\title{
Localized interface modes in one-dimensional hyperuniform acoustic materials
}

\author{
S.M. Kuznetsova \\ Laboratoire d'Acoustique de l'Université du Mans (LAUM), UMR CNRS 6613, \\ Institut d'Acoustique-Graduate School (IA-GS), CNRS, Le Mans Université, Avenue \\ Olivier Messiaen, 72085 Le Mans, France \\ E-mail: smkuznetsova1@gmail.com
}

\section{J.-P. Groby}

Laboratoire d'Acoustique de l'Université du Mans (LAUM), UMR CNRS 6613, Institut d'Acoustique-Graduate School (IA-GS), CNRS, Le Mans Université, Avenue Olivier Messiaen, 72085 Le Mans, France

\section{M. García-Raffi}

Instituto Universitario de Matemática Pura y Aplicada (IUMPA), Universitat Politècnica de València, Camino de vera s/n 46022 València, Spain

\section{Romero-García}

Laboratoire d'Acoustique de l'Université du Mans (LAUM), UMR CNRS 6613, Institut d'Acoustique-Graduate School (IA-GS), CNRS, Le Mans Université, Avenue Olivier Messiaen, 72085 Le Mans, France

December 2020

\begin{abstract}
We theoretically, numerically and experimentally report the localization of an acoustic wave at the interface between two one-dimensional hyperuniform materials of different geometrical representations. These materials suppress the acoustic scattering in the long wavelength regime, being rather disordered and degenerate, while possessing a wide band gap. In this work, these hyperuniform materials are made of an air-filled acoustic waveguide with rigid diaphragms acting as scatterers. A wide band gap and the emergence of the edge modes provide promising applications in wave control devices.
\end{abstract}

\section{Introduction}

Localized edge modes occurring at the connection between two materials yielding in different topological phases have long attracted attention due to their potential applications for robust transport of different types of waves. The eigenfrequencies of such boundary modes lie inside the band gap flanked by allowed bands. Translational 
symmetry is of crucial importance for both the existence of the band gaps and the introduction of bulk topological invariants of the system. In this regard, the topological properties are traditionally attributed to ordered materials. The emergence of the boundary modes is governed by the bulk-boundary correspondence, which is a relation between the eigenstates of the system within the bulk spectrum and the number of the supported interface modes. These modes are topologically protected, i.e., they are stable against adiabatic chiral symmetry preserving perturbations if the band gap remains open [22, 8]. The topological state of the system can be controlled by the gap closing and reopening, which is driven by the geometrical parameters.

One of the most established and common one-dimensional (1D) systems to describe topological edge states is the Su-Schrieffer-Heeger ( $\mathrm{SSH}$ ) model [27]. This model is widely used to predict and investigate the localized boundary modes in binary waveguide arrays [6], diatomic chains of plasmonic particles [16], dielectric microwave resonators [24], spins [11, etc. Topology has recently enriched the fields of acoustics and mechanics by introducing various classical analogs of quantum and electronic effects, such as Dirac cone dispersion, quantum Hall and spin Hall effects [17, 21, 33. Periodic acoustic structures have been shown to undergo a topological phase transition accompanied by the emergence of the edge localized modes by means of varying the geometry of the sample [31, 17, 23, 10, 15, 20. In particular, the variation of the lengths of the unit cell components has been shown to affect the topological properties of the second [31] and higher order gaps [20] in one-dimensional systems.

Meanwhile, topologically nontrivial states have been achieved not only in periodic structures. Topological edge modes have been observed in quasiperiodic structures [2], coupled resonator smartly patterned systems [3] and even in amorphous systems with randomly distributed particles [1, 9].

Lately, hyperuniform materials have emerged as promising candidates for the wave control, exhibiting transparency for a set of wavevectors in the long wavelength limit. Hyperuniform structures stand apart from conventional media exhibiting the properties of liquids (amorphous) and crystals (periodic) simultaneously - suppressing large scale density fluctuations. They are statistically isotropic with no long-range order [30, 29, 5]. They were found to possess wide isotropic bandgaps both in photonic [19], [18] and phononic [13] systems, in spite of being highly disordered and degenerate. These materials thus offer remarkable capabilities for engineering waveguiding devices. The band gap formation is attributed to the interplay of the hyperuniformity and the reminiscences of crystallinity always present due to the constraints imposed on the system [12]. Since the band gap closing and reopening can be achieved by varying the geometric parameters of the hyperuniform material similarly to the periodic systems, the crucial changes in the bulk eigenstates can be undergone.

In this work, we theoretically and numerically predict and experimentally observe the wave localization at the interface between two hyperuniform materials both possessing a band gap in similar ranges of frequencies. This counter-intuitive result shows the possibility of localizing waves in a controlled manner between two disordered 
systems due to the reminiscence of periodicity in the hyperuniform materials. To obtain a hyperuniform structure, we utilize the optimization procedure described in Ref. [25]. The extracted hyperuniform point distribution is used to manufacture a 1D hyperuniform material made of an air-filled waveguide of circular cross-section in which the hyperuniform distribution of rigid diaphragms is embedded. Wave propagation is numerically analyzed and experimentally validated showing good agreement. By changing the geometry of the diaphragms, the band gap closing and reopening is achieved and two complementary configurations at the opposite sides of the band gap closing are obtained. These two systems are then connected to analyze the possible localization of the acoustic wave at the interfaces between these two hyperuniform media. We also revisit the periodic counterpart of the localized edge states in periodic media from a theoretical and experimental points of view.

\section{Hyperuniform materials}

Consider a 1D distribution of $N$ identical scatterers located at positions $x_{j}$ which form a unit cell of size $L$ that is periodically repeated along the $x$-coordinate. The reciprocal counterpart of this complex lattice is given by the reciprocal lattice vector $G=2 \pi m / L$, $m \in \mathbb{Z}$. In case of negligible interactions between the scatterers, such systems can be described by the structure factor $S(G)$

$$
S(G)=\frac{1}{N}\left|\sum_{j=1}^{N} e^{i G x_{j}}\right|^{2} .
$$

The system is hyperuniform if the long-range density fluctuations are suppressed leading to vanishing structure factor $S(|G|<K)=0$ in the vicinity of the origin of the reciprocal space with $K=2 \pi n / L, n \in \mathbb{N}$. The hyperuniform patterns are also characterized by the parameter $\chi$, which defines a relative number of the independent reciprocal lattice vectors lying in the region $|G|<K$. For 1 D systems, $\chi=n / N$. When $\chi \geq 0.5$, the pattern becomes crystalline, because crystal becomes the only way to meet the requirement of minimum value of the structure factor [12]. In the limit $\chi \rightarrow 0$, the configurations are disordered and represent an ideal gas, since there is no constraint anymore on the reciprocal lattice vectors [30]. In the intermediate regime, $0<\chi<0.5$ the configurations are disordered, although they still display some hints of crystallinity,

such as reminiscences of the Bragg peaks [12] leading to isotropic gaps in the transmission spectrum. Thus, the introduction of $\chi$ as an order measure illustrates that states of matter exist between crystals and ideal gases with counter-intuitive physical properties.

To design the hyperuniform pattern, we use an optimization procedure, which looks for the positions of point scatterers $x_{j}$ that minimize the structure factor for a target region $|G|<K$ as described in Ref. [25]. The algorithm provides a certain configuration starting from a random distribution satisfying the constraint that the particles cannot overlap $\left(\left|x_{i}-x_{j}\right| \geq l\right.$ with $l$ being the size of the particle), so that the 


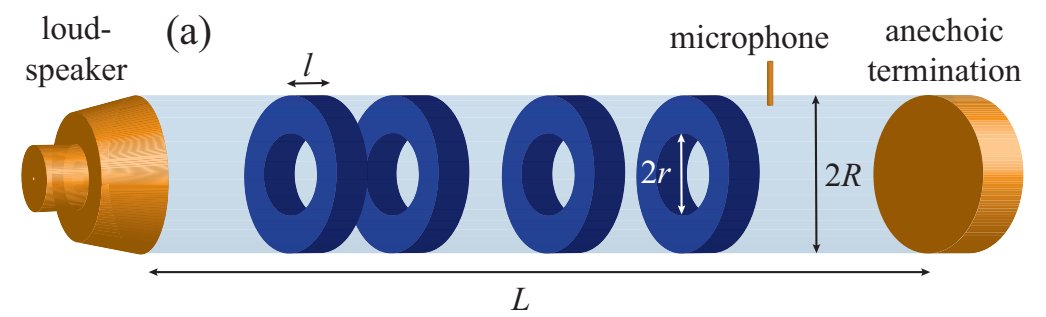

(b)

(c)

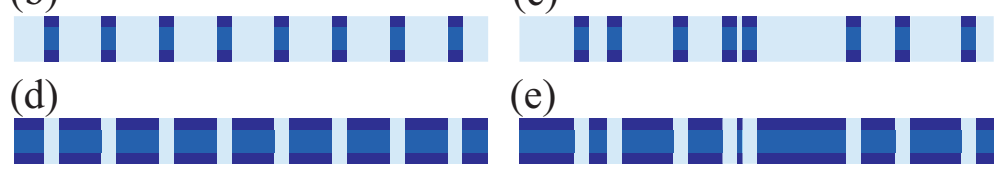

Figure 1. (a) Schematic view of the experimental setup, (b), (d) longitudinal section of the positive and negative periodic waveguides respectively, (c), (e) longitudinal section of the positive and negative hyperuniform waveguides respectively. The dark blue regions correspond to the diaphragms and the light blue regions correspond to the empty spaces inside the waveguide.

aposteriori experimental validation should be performed. The objective functions to be simultaneously minimized are the structure factor

$$
\phi\left(x_{1}, \ldots x_{N}\right)=\sum_{|G|<K} S(G),
$$

and the standard deviation function

$$
\sigma=\sqrt{\frac{1}{N-1} \sum_{|G|<K}\left|S(G)-\frac{1}{N} \sum_{|G|<K} S(G)\right|^{2}} .
$$

We focus on the single unit supercell of the hyperuniform sample with $\chi=0.2$ to ensure a high degree of disorder in the structure. Studying a single unit supercell is sufficient to represent a hyperuniform material in 1D systems. With such moderate value of $\chi$, the area of suppressed structure factor $(f \leq 680 \mathrm{~Hz})$ is small and the material is closer to a disordered material than to a periodic one. Nevertheless, the constraint on the minimum distance between the scatterers introduces a short-range correlation and leads to the reminiscences of crystallinity of the system, which will be discussed in the following sections.

\section{Experimental setup}

The setup utilized in this work is made of a main waveguide of radius $R$ in which diaphragms with inner radius $r<R$ act as the scatterers of the system. $N$ diaphragms located at the specific positions provided by the optimization procedure will constitute the whole system (see Appendix A for the exact positions). A scheme of the setup is shown in Fig. 1. The dimensions are chosen to satisfy the single mode propagation regime in the frequency range of interest, i.e., the range of frequencies analyzed in the 
setup are always smaller than the cutoff frequency of the waveguide. The radius of the waveguide is $R=1.5 \mathrm{~cm}$ and its length is $L=1 \mathrm{~m}$. The hyperuniform material supercell is optimized considering $N=20$ scatterers. The radii of the diaphragms are chosen identical $r=R / 2$ in order to open the band gap of the hyperuniform material. A plexiglass tube and PVC diaphragms were used in the experiments. Both materials are considered acoustically rigid due to their large impedance mismatch with respect to that of the air. A loudspeaker was used to generate a plane wave at one end of the plexiglass tube and a single microphone was used to measure the amplitude of the pressure field at the desired locations. At the opposite end of the tube, an anechoic termination with less than $5 \%$ of reflection amplitude in the analyzed frequency range was used.

In this work, we consider either a periodic or a hyperuniform distributions of scatterers represented by two configurations residing in different states. We introduce the variable radii $r^{\prime}(t)=R / 2(1+t), R^{\prime}(t)=R(1-t / 2)$ and the parameter $t \in[0,1]$

for the simulation of the band gap closing and reopening. Two complementary configurations referred to as the positive and the negative configurations are obtained by varying $t$ from 0 to 1 (see Figs. 1(c),(e)). They stay at different states, i.e., the positive configuration stays on the left of the band gap closing with $t<0.5$ while the negative one stays on the right of the band gap closing with $t>0.5$.

In practice, the positive configuration is realized by locating the diaphragms of length $l=1.5 \mathrm{~cm}$ at the scatterers positions of the hyperuniform point pattern, while the negative configuration is realized by locating the different diaphragms between the scatterers positions. These two configurations with $t=0$ and $t=1$ are used in the experiments.

The same concept is applied to the periodic system. The positive and the negative periodic waveguides with 5 inserted diaphragms are considered. Two types of diaphragms were fabricated - the first type of lengths $l_{2}=1.5 \mathrm{~cm}$ and the second type of lengths $l_{2}=3.5 \mathrm{~cm}-$ for the construction of the periodic waveguides. The size of the constituent unit cell in both configurations is fixed to $d=l_{1}+l_{2}=5 \mathrm{~cm}$. In this way, the two configurations are complementary to each other (see Figs. 1(b),(d)). The periodic structure made of the diaphragms of length $l_{2}=1.5 \mathrm{~cm}$ is referred to as positive configuration, while that made of the diaphragms of length $l_{2}=3.5 \mathrm{~cm}$ is referred to as the negative configuration.

\section{Revising the localized modes in 1D periodic systems}

\subsection{Characteristics of $1 D$ periodic materials}

A 1D crystalline scatterers distribution is implemented by periodically embedding diaphragms of radii $r=R / 2$ and length $l_{2}$ acting as scatterers in an air-filled waveguide of radius $R$ (Fig. 2(a)). Only plane acoustic waves are assumed to propagate.

The pressure $p$ and the acoustic flow $u$ at 2 points (initial $i$ and final $f$ ) along the 
waveguide are related via the transfer matrix $T$

$$
\left(\begin{array}{l}
p_{i} \\
u_{i}
\end{array}\right)=T\left(\begin{array}{l}
p_{f} \\
u_{f}
\end{array}\right),
$$

where $T$ is the product of all the intermediate transfer matrices along the $i \rightarrow f$ path

$$
T=\prod_{j=1}^{N} T_{j}
$$

The transfer matrix of a waveguide or a diaphragm of the length $l_{j}$, cross section $S_{j}$ and reduced impedance $Z_{j}=\rho c / S_{j}$ is

$$
T_{j}=\left(\begin{array}{cc}
\cos \left(k l_{j}\right) & \imath Z_{j} \sin \left(k l_{j}\right) \\
\frac{\imath}{Z_{j}} \sin \left(k l_{j}\right) & \cos \left(k l_{j}\right)
\end{array}\right),
$$

where $k$ is a wavenumber and the $e^{-i \omega t}$ time harmonic dependence is assumed. The flow in the final point is obtained from Eq. (4)

$$
u_{f}=V_{i, f} p_{f}+W_{i, f} p_{i},
$$

where $V_{i, f}=-\frac{T_{11}}{T_{12}}, W_{i, f}=\frac{1}{T_{12}}$. Considering the acoustic flows from the points $(m+1)$ and $(m-1)$ into the point $m$, we obtain

$$
\left(V_{m-1, m}+V_{m+1, m}\right) p_{m}=-W_{m-1, m} p_{m-1}-W_{m+1, m} p_{m+1} .
$$

The opposite signs of flows coming from opposite directions (from the points $m+1$ and $m-1$ ) into the point $m$ have been accounted for.

Let us refer to the junctions with a large radius part at the right as the A-junctions and those with a large radius part at the left as the B-junctions (Fig. 2(b)). If $l_{1}=l_{2}=l$, Eq. (8) takes the form of a pair of Su-Schrieffer-Heeger ( $\mathrm{SSH}$ ) equations

$$
\begin{aligned}
& \varepsilon A_{n}=\kappa_{2} B_{n-1}+\kappa_{1} B_{n}, \\
& \varepsilon B_{n}=\kappa_{2} A_{n+1}+\kappa_{1} A_{n},
\end{aligned}
$$

where $A_{n}, B_{n}$ represent the pressures at the corresponding junctions, $\varepsilon=\cos (k l)$, $\kappa_{1}=\frac{R^{2}}{r^{2}+R^{2}}$, and $\kappa_{2}=\frac{r^{2}}{r^{2}+R^{2}}$. The junctions are now identified to the particles in a dimer chain of the original SSH model with a unit cell $[A B]$. According to the expressions of the coupling coefficients $\kappa_{1,2}$, the parts of the tube with radii $R$ and $r$ correspond to the strong and weak couplings between the particles. Thus, the region between the junctions $A_{n}$ and $B_{n}$ of a waveguide plays the role of the unit cell and the region between the junctions $B_{n}$ and $A_{n+1}$ plays the role of the connection between the unit cells. Another unit cell can be chosen, as that depicted in Fig. 2(c), which would lead to the same system of equation but with interchanged coupling coefficients $\kappa_{1}$ and $\kappa_{2}$.

The dispersion relation of the SSH system exhibits a gap $\Delta \varepsilon=\left|\kappa_{1}-\kappa_{2}\right|$. When $\kappa_{1}=\kappa_{2}$ the gap closes and the eigenstates of the system with arbitrarily small eigenvalues are allowed propagating modes. The condition $\kappa_{1}=\kappa_{2}$ corresponds to $R=r$, i.e., the empty tube. If the system is finite $(n=1, \ldots, N)$, it may support zero-eigenvalue states. Assuming $\varepsilon=0$ in Eq. (9), the solution becomes 




Figure 2. (a) Scheme of a 1-D waveguide of radius $R$ with diaphragms of radius $r$ and length $l_{2},(\mathrm{~b}),(\mathrm{c})$ corresponding SSH-like chains of point particles of type A and $\mathrm{B}$ with different choices of a unit cell $[A B]$.

$$
\begin{aligned}
& \left(\begin{array}{l}
A_{n} \\
B_{n}
\end{array}\right)=(-1)^{n-1}\left(\frac{\kappa_{1}}{\kappa_{2}}\right)^{n-1}\left(\begin{array}{c}
A_{1} \\
0
\end{array}\right), \\
& \left(\begin{array}{c}
A_{n} \\
B_{n}
\end{array}\right)=(-1)^{n-1}\left(\frac{\kappa_{2}}{\kappa_{1}}\right)^{n-1}\left(\begin{array}{c}
0 \\
B_{1}
\end{array}\right) .
\end{aligned}
$$

Having an eigenfrequency inside the band gap, the solutions should be localized at the sites $A_{1}$ and $B_{N}$. This requirement is fulfilled if the inter-cell coupling $\kappa_{2}$ is stronger than the intra-cell one $\kappa_{1}$. Otherwise, the solutions are delocalized and should be disregarded. The localized edge states are topologically protected. They remain present under the adiabatic modifications of the parameters (continuous modifications preserving the chiral symmetry inherent to this system [4] and the open band gap). The case $\kappa_{2}>\kappa_{1}$ is qualified as topological, while the opposite one is trivial.

Now, we consider the waveguide with different lengths of the diaphragms and the empty spaces $\left(l_{1} \neq l_{2}\right)$. The system is thus described by modified equations. However, the chiral symmetry is preserved and the system presents the features similar to those of the SSH model in terms of the band structure and emergence of interface states.

The localized edge states appear not only at the edges of the chain, but also at the interface between the two phases - the trivial and topological ones. There are two ways to connect two finite structures of different topological phases - via weak (Fig. 3(a)) or strong (Fig. 3(b)) coupling [6]. The former is a monomer defect and the later is a trimer defect, represented by a particle $A$ and particles $A B A$ written in red in Figs. 3(a),(b) respectively. When $l_{1}=l_{2}$ a monomer defect supports a localized mode with a maximum amplitude at the defect particle $A$, while the trimer defect supports a localized mode with a node at the defect center $B[6,10]$. When $l_{1} \neq l_{2}$ the similar behaviour of the interface modes is expected. 
(a)
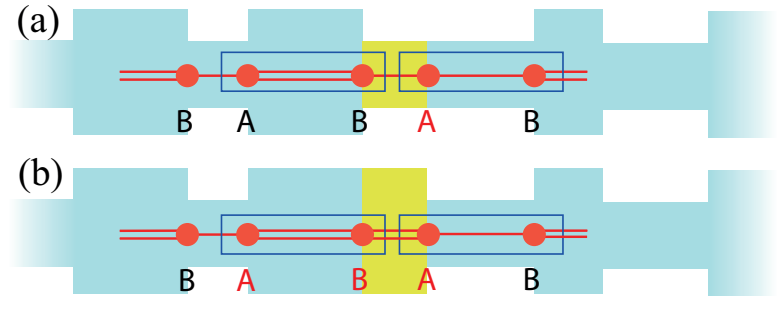

Figure 3. (a) a monomer and (b) a trimer defects between two periodic waveguides and the corresponding SSH-like particle chains.

\subsection{Observation of the localized interface modes in periodic systems}

We consider a $1 \mathrm{D}$ crystalline scatterers distribution with $l_{1} \neq l_{2}$. In Fig. 4(a), we plot the dispersion relation of the positive (red dots) and the negative (blue dots) configurations. If $R$ and $r$ are continuously interchanged, the band gap closes and reopens (see the inset in Fig. 4(a), where the lower and upper edge bands of the band gap of the different configurations at $d k=\pi$ are represented). The transmission coefficients of these finite configurations are plotted in Figs. $4(\mathrm{~b})$ and (c). The region with transmission suppression corresponds to the position of the band gap predicted by the dispersion relation. The equidistant peaks outside the band gap are attributed to the Fabry-Perot resonances of a finite waveguide.

Since the connection of two materials in different topological phases supports a localized interface mode in the SSH model, we expect the same behavior in our periodic structures. In order to analyze the effect of the localized modes in the dispersion relation, we have to consider a new supercell made of the combination of a positive and a negative supercells in which the defect is in the middle, as it is usually done in the literature $([6,23])$. Thus we consider a supercell consisting of the connection of a positive and a negative supercells with 5 periods in each, being enough distance to avoid the coupling between the localized modes due to the periodicity of the supercell. Indeed, two localized modes inside a band gap with a flat dispersion, corresponding to two types of connection between the waveguides - weak and strong ones - are encountered in the dispersion diagram depicted in Fig. 5(a). The weak connection supports a lower frequency mode, with a symmetric pressure profile relative to the connection point. The strong connection maintains a higher frequency mode with an antisymmetric profile. In Fig. 5(b), we plot the normalized pressure amplitudes at the connection between the two waveguides coupled in the two described ways: red (blue) lines and symbols correspond to the strong (weak) connection supporting an antisymmetric (a symmetric) mode. In the insets of Fig. 5(b), we plot the pressure field distributions (the real parts) of the interface modes showing the symmetry of each interface mode. The experimental measurements are shown as circles and appear to be in a good agreement with the predictions. The black lines represent the expected interface modes frequencies from the dispersion diagram that match the experimental peaks.

Surface modes at the interface between two materials of surface impedances $Z_{1}$ 


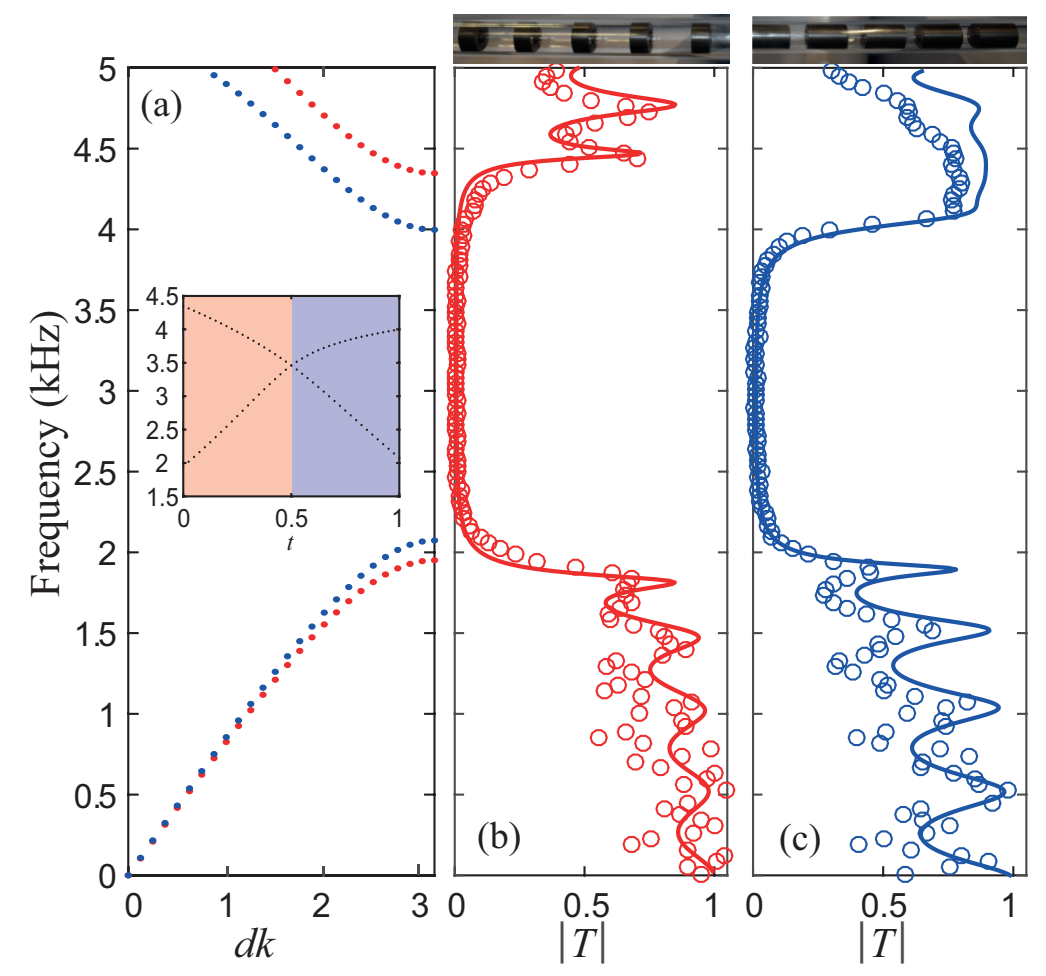

Figure 4. (a) Dispersion diagram of a periodic waveguide in positive (red dotted line) and negative (blue dotted line) configurations, the band gap closing and reopening is shown on the inset, (b),(c) transmission coefficients of the positive and negative cases respectively. The solid lines correspond to the calculations and the circles correspond to the experimental results.

and $Z_{2}$ appear as the poles of the reflection coefficient $R_{12}=\frac{Z_{1}-Z_{2}}{Z_{1}+Z_{2}}$. For photonic [14] and phononic [32, 20] crystals, $Z_{1,2}$ are related to the topological invariants of the corresponding materials. The equality

$$
Z_{1}+Z_{2}=0
$$

is thus a condition for the emergence of the topologically protected interface states. Different signs of the surface impedances indicate that the two connected materials reside in different topological phases. The surface impedance of the material can be obtained by measuring the reflection coefficient at its interface with air $Z_{1,2}=Z_{0} \frac{1+R_{1,2}}{1-R_{1,2}}$, where $Z_{0}$ is the impedance of air. Thus, the equation Eq. (12) reduces to the requirement [32, 20]

$$
\begin{aligned}
& \Re\left(R_{1} R_{2}\right)=1, \\
& \Im\left(R_{1} R_{2}\right)=0 .
\end{aligned}
$$

Figure 5(c) illustrates the product of the reflection coefficients from the single positive and negative waveguides $R_{p} R_{n}$ at the ends, which form a strong (read line) and a weak (blue line) connections. The frequencies satisfying Eq. (13) perfectly match the frequencies of the localized modes identified in the dispersion diagram (black dotted lines). Thus, the red and blue regions on the inset in Fig. 4(a) correspond to different topological phases. 

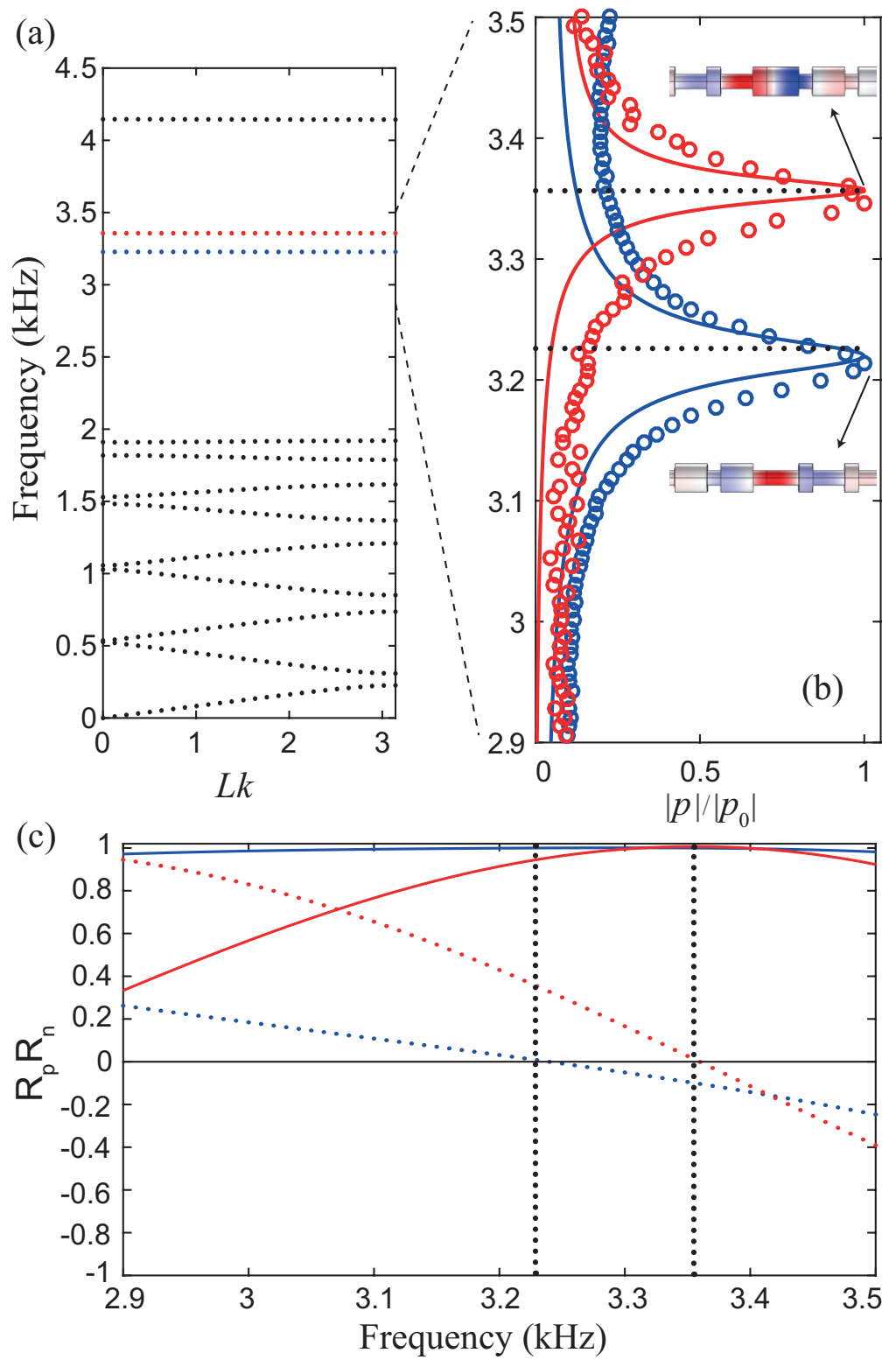

Figure 5. (a) Dispersion diagram of connected positive and negative periodic waveguides, (b) normalized pressure amplitude at the connection between the positive and negative waveguides measured with respect to the amplitude inside an empty tube $p_{0}$. The red line corresponds to the strong connection and the blue line corresponds to the weak connection. The circles represent the experimental results. The insets represent pressure distributions in the corresponding modes, (c) $\operatorname{Re}\left(R_{p} R_{n}\right)$ (solid lines) and $\operatorname{Im}\left(R_{p} R_{n}\right)$ (dotted lines) as functions of frequency for strong (red) and weak(blue) connection of two periodic waveguides. Black dotted lines correspond to the expected frequencies from the dispersion diagram. 


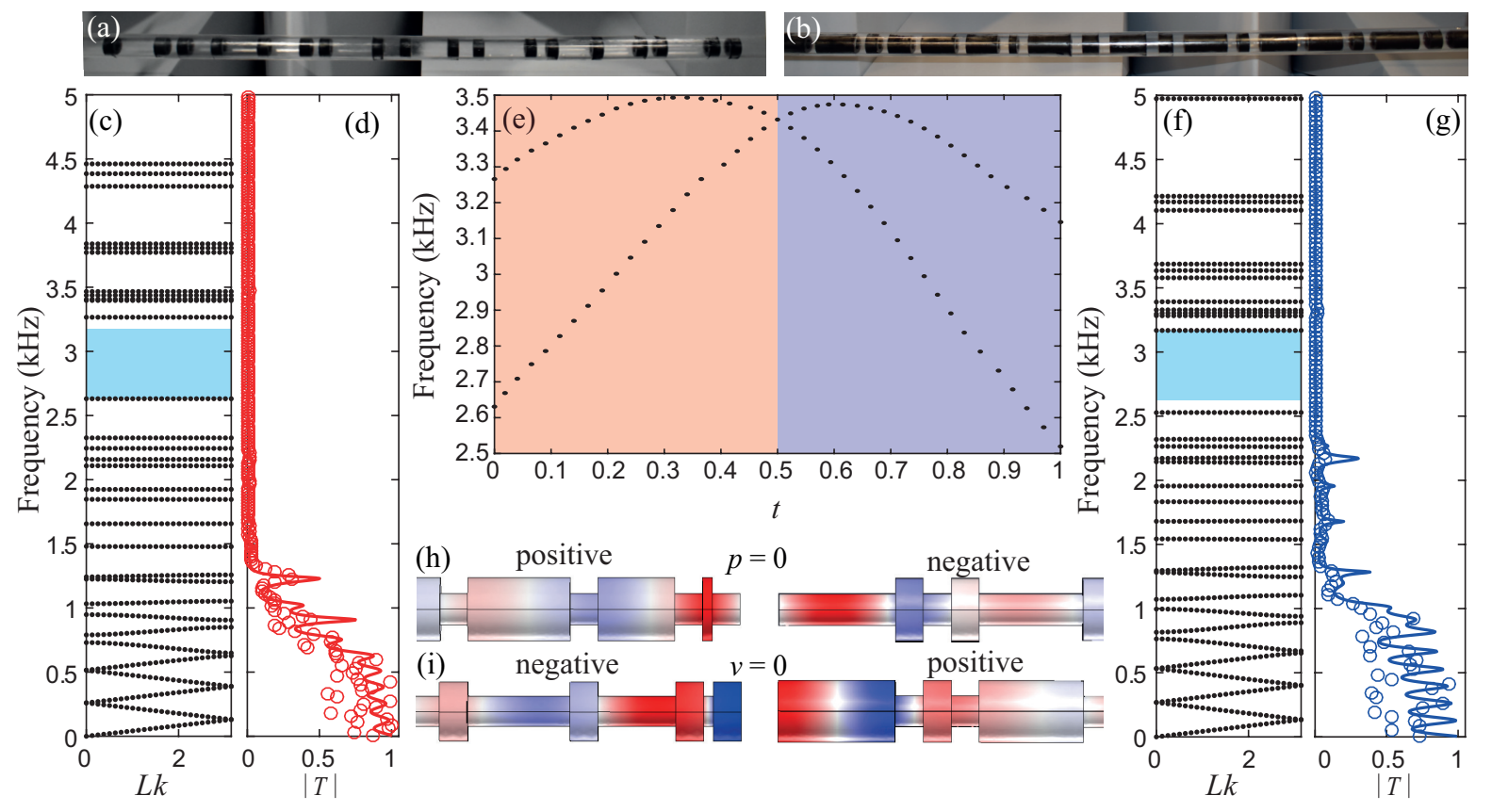

Figure 6. (a) positive and (b) negative waveguides designed upon a hyperuniform scatterers distribution, (c),(d) dispersion diagram and the transmission coefficient of a positive waveguide, (e) closing of the band gap of a hyperuniform material with the variation of the waveguide and diaphragms radii, the red region corresponds to the positive waveguide, the blue region corresponds to the negative one, (f), (g) dispersion diagram and the transmission coefficient of a negative waveguide, (h),(i) pressure profiles in the edge modes of single positive and negative waveguides for Dirichlet and Neumann boundary conditions.

\section{Properties of the hyperuniform materials: Closing and reopening band gap}

\subsection{Characteristics of the 1D Hyperuniform materials}

We first analyze both the dispersion relation and the transmission through the system. Figure 6(a) shows the distribution of scatterers in the designed positive hyperuniform material and Fig. 6(c) depicts its dispersion relation. Several flat eigenmodes, attributed to the modes localized inside the waveguide due to the disordered distribution of scatterers, are located in the band gap. These modes present a very low dispersion with small group velocity. The presence of the viscothermal losses in the system dramatically impacts them [28]. This results in a very low transmission coefficient over a broad frequency range, as shown in Fig. 6(d). This remarkable transmission dip is much wider than that of a periodic waveguide studied in the 4 . The peaks in the transmission coefficients in the low frequency propagative regime are attributed to Fabry-Perot resonances (see Appendix B).

We now focus on the blue region highlighted in the dispersion diagram in Fig. 6(c). It corresponds to the gap between the $20^{\text {th }}$ and $21^{\text {st }}$ modes, where a periodic structure consisting of 20 unit cells possesses a band gap due to the folding of the Brillouin zone. 
Thus, we expect to observe some reminiscence of the periodicity in this region. The width of this band gap is defined by the ratio of the radius of the diaphragm over that of the waveguide, as it can be seen from the Fig. 6(e), where the lower and upper edge of the band gap at $L k=0$ represented by the blue area in Fig. 6(c) are depicted. The positive and the negative configurations are shown in Figs. 6(a),(b) respectively. The dispersion diagram and the transmission spectrum of the negative configuration shown in Figs. 6(f), (g) respectively are similar to those of the positive one. The band gap we are interested in, shown by the blue region, lies in the same frequency range as for the positive configuration.

\subsection{Observation of the localized edge states in hyperuniform materials}

We have revised the features of periodic structures as well as theoretically and experimentally shown in Sec. 4 the excitation of edge modes by using periodic distribution of scatterers in our setup. In this work, although we study a disordered system, periodicity related features still remain. Thus, we expect to observe the reminiscences of the topologically protected states at the interface between a positive and a negative hyperuniform materials. To do that we proceed as in the periodic case by considering a supercell made of the connection of a positive and a negative supercells allowing us to analize the effect of the localized modes in the dispersion relation.

Both positive and negative waveguides under consideration possess localized edge modes with frequencies inside the band-gap. Their profiles are shown in Figs. 6(h),(i) for the two types of boundary condition - Dirichlet $(p=0)$ and Neumann $(v=0)$ respectively.

The positive and negative waveguides can be connected in two ways - either by means of the ends of a larger radius (referred to as the strong connection), or by means of the ends of a smaller radius (referred to as the weak connection). We notice the localized interface modes inside the band gap, as shown in the dispersion relation in Fig. 7(a). The mode profiles are shown on the insets of Fig. 7(b) next to the corresponding normalized pressure amplitudes measured at the connection. The numerical prediction (continuous line) and the experimental results (symbols) are in good agreement. The reminiscences of the symmetric and antisymmetric behavior of $\operatorname{Re}(p)$ of the field of these modes, which is inherent in the periodic case, are visible. The frequencies of the interface modes are in good agreement with the predictions of the dispersion relation.

We evaluated numerically the reflection coefficients of the single positive $\left(R_{p}\right)$ and negative $\left(R_{n}\right)$ hyperuniform materials at their right and left ends, in order to account for both types of connections, considering a single unit supercell. Due to the fact that we are in the band gap region, that we are considering a $1 \mathrm{D}$ system, and that at this particular frequency the reflection coefficient is maximal, the recovered impedance is that of the semi-infinite medium. The scattering coefficients are obtained from the pressure evaluated at two points upstream and downstream the material[26]. The real and imaginary parts of $R_{p} R_{n}$ are shown in Fig. 7)(c) in solid and dotted lines respectively. The frequencies, where the Eq. 13) is satisfied, perfectly match those of 



Figure 7. (a) dispersion diagram of connected positive and negative hyperuniform waveguides, (b) normalized pressure amplitude at the connection between the positive and negative waveguides measured with respect to the amplitude inside the empty tube $p_{0}$. The red line corresponds to the strong connection and the blue line corresponds to the weak one, the circles represent the experimental results, (c) $\operatorname{Re}\left(R_{p} R_{n}\right)$ (solid lines) and $\operatorname{Im}\left(R_{p} R_{n}\right)$ (dotted lines) for strong (red) and weak(blue) connection. Black dotted lines correspond to the expected frequencies from the dispersion diagram.

the localized modes in the dispersion diagram (vertical black dotted lines). Thus, the red and blue regions in Fig. 6(e) correspond to the reminiscences of different topological phases inherent to periodic structures. 


\section{Conclussion}

In this work we have implemented a previously developed approach [25] to engineer a 1D hyperuniform material, which possesses extremely broad band transmission suppression. The system was analyzed numerically and experimentally and consists in a waveguide with rigid diaphragms embedded in. Interchanging the locations of the diaphragms and the empty spaces, we reach a transition point, which resembles the topological phase transition occurring in the periodic systems. Despite the system is disordered, connecting two waveguides yielding on opposite sides from this point leads to an emergence of localized interface modes. Their profiles are similar to those of the periodic waveguides. Thus, we observe the reminiscences of non-trivial topological behavior of periodic structures in the disordered system.

\section{Acknowledgments}

This work has been funded by the project HYPERMETA funded under the program Étoiles Montantes of the Région Pays de la Loire as well as by the ANR-RGC METARoom (ANR-18-CE08-0021) project. 



Figure B1. Transmission coefficient, dispersion relationand and the pressure profiles (real parts) of the low-frequency Fabry-Perot resonances of the positive hyperuniform waveguide.

\section{Appendix A. Diaphragms positions in a hyperuniform waveguide}

Positions of the left edges of the diaphragms in the positive hyperuniform configuration

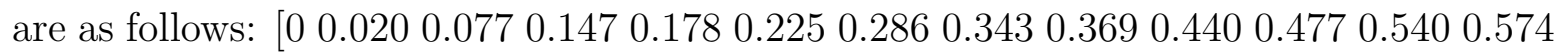
$\begin{array}{lllllll}0.644 & 0.673 & 0.734 & 0.793 & 0.820 & 0.892 & 0.922]\end{array}$ (m).

\section{Appendix B. Low-frequency Fabry-Perot resonances}

We have performed a calculation of the transmission coefficient $T$ of the lossless positive hyperuniform material, which is shown in the Fig. B1 together with the dispersion diagram. The low-frequency peaks are equidistant and every following peak provides a pressure profile with an additional node as compared to the previous one (the right panel of the figure). These are the properties of the Fabry-Perot resonances. In addition, from the dispersion diagram one can calculate the velocity of approximately $260.7 \mathrm{~m} / \mathrm{s}$. Inserting this value into the condition of the Fabry-Perot resonances $L k=n \pi$ one obtains a resonance frequency difference of approximately $130.35 \mathrm{~Hz}$, which equals to the distance between the low-frequency peaks. As the speed of sound reduces with the increase of frequency due to the dispersion, the higher-frequency peaks are farther apart. The modes corresponding to the peaks (f) and (h) are attenuated because of their location inside a band gap. The higher-order modes (i) and (j) are localized inside the waveguide and have a rather small velocity corresponding to the localized modes. 


\section{References}

[1] A. Agarwala and V. B. Shenoy. Topological insulators in amorphous systems. Phys. Rev. Lett., 118:236402, Jun 2017.

[2] D. J. Apigo, W. Cheng, K. F. Dobiszewski, E. Prodan, and C. Prodan. Observation of topological edge modes in a quasiperiodic acoustic waveguide. Phys. Rev. Lett., 122:095501, Mar 2019.

[3] D. J. Apigo, K. Qian, C. Prodan, and E. Prodan. Topological edge modes by smart patterning. Phys. Rev. Materials, 2:124203, Dec 2018.

[4] J. Asbóth, L. Oroszlány, and A. Pályi. A Short Course on Topological Insulators: Band Structure and Edge States in One and Two Dimensions. Springer International Publishing, 2016.

[5] R. D. Batten, F. H. Stillinger, and S. Torquato. Classical disordered ground states: Super-ideal gases and stealth and equi-luminous materials. Journal of Applied Physics, 104(3):033504, 2008.

[6] A. Blanco-Redondo, I. Andonegui, M. J. Collins, G. Harari, Y. Lumer, M. C. Rechtsman, B. J. Eggleton, and M. Segev. Topological optical waveguiding in silicon and the transition between topological and trivial defect states. Phys. Rev. Lett., 116:163901, Apr 2016.

[7] C. Brendel, V. Peano, O. Painter, and F. Marquardt. Snowflake topological insulator for sound waves. arXiv preprint arXiv:1701.06330, 2017.

[8] B.-H. Chen and D.-W. Chiou. An elementary rigorous proof of bulk-boundary correspondence in the generalized su-schrieffer-heeger model. Physics Letters A, 384(7):126168, 2020.

[9] M. Costa, G. R. Schleder, M. Buongiorno Nardelli, C. Lewenkopf, and A. Fazzio. Toward realistic amorphous topological insulators. Nano letters, 19(12):8941-8946, 2019.

[10] M. Esmann, F. R. Lamberti, A. Lemaître, and N. D. Lanzillotti-Kimura. Topological acoustics in coupled nanocavity arrays. Phys. Rev. B, 98:161109, Oct 2018.

[11] M. P. Estarellas, I. D'Amico, and T. P. Spiller. Topologically protected localised states in spin chains. Scientific reports, 7(1):1-10, 2017.

[12] Y. Fan, J. K. Percus, D. K. Stillinger, and F. H. Stillinger. Constraints on collective density variables: One dimension. Phys. Rev. A, 44:2394-2402, Aug 1991.

[13] G. Gkantzounis, T. Amoah, and M. Florescu. Hyperuniform disordered phononic structures. Phys. Rev. B, 95:094120, Mar 2017.

[14] F. J. Lawrence, L. C. Botten, K. B. Dossou, R. C. McPhedran, and C. M. de Sterke. Photoniccrystal surface modes found from impedances. Phys. Rev. A, 82:053840, Nov 2010.

[15] X. Li, Y. Meng, X. Wu, S. Yan, Y. Huang, S. Wang, and W. Wen. Su-schrieffer-heeger model inspired acoustic interface states and edge states. Applied Physics Letters, 113(20):203501, 2018.

[16] C. W. Ling, M. Xiao, C. T. Chan, S. F. Yu, and K. H. Fung. Topological edge plasmon modes between diatomic chains of plasmonic nanoparticles. Opt. Express, 23(3):2021-2031, Feb 2015.

[17] G. Ma, M. Xiao, and C. Chan. Topological phases in acoustic and mechanical systems. Nature Reviews Physics, 1(4):281-294, 2019.

[18] W. Man, M. Florescu, K. Matsuyama, P. Yadak, G. Nahal, S. Hashemizad, E. Williamson, P. Steinhardt, S. Torquato, and P. Chaikin. Photonic band gap in isotropic hyperuniform disordered solids with low dielectric contrast. Optics express, 21(17):19972-19981, 2013.

[19] W. Man, M. Florescu, E. P. Williamson, Y. He, S. R. Hashemizad, B. Y. C. Leung, D. R. Liner, S. Torquato, P. M. Chaikin, and P. J. Steinhardt. Isotropic band gaps and freeform waveguides observed in hyperuniform disordered photonic solids. Proceedings of the National Academy of Sciences, 110(40):15886-15891, 2013.

[20] Y. Meng, X. Wu, R.-Y. Zhang, X. Li, P. Hu, L. Ge, Y. Huang, H. Xiang, D. Han, S. Wang, and W. Wen. Designing topological interface states in phononic crystals based on the full phase diagrams. New Journal of Physics, 20(7):073032, jul 2018.

[21] S. H. Mousavi, A. B. Khanikaev, and Z. Wang. Topologically protected elastic waves in phononic metamaterials. Nature communications, 6(1):1-7, 2015.

[22] T. Ozawa, H. M. Price, A. Amo, N. Goldman, M. Hafezi, L. Lu, M. C. Rechtsman, D. Schuster, J. Simon, O. Zilberberg, and I. Carusotto. Topological photonics. Rev. Mod. Phys., 91:015006, 
Mar 2019.

[23] R. K. Pal, J. Vila, M. Leamy, and M. Ruzzene. Amplitude-dependent topological edge states in nonlinear phononic lattices. Phys. Rev. E, 97:032209, Mar 2018.

[24] C. Poli, M. Bellec, U. Kuhl, F. Mortessagne, and H. Schomerus. Selective enhancement of topologically induced interface states in a dielectric resonator chain. Nature communications, 6(1):1-5, 2015.

[25] V. Romero-García, N. Lamothe, G. Theocharis, O. Richoux, and L. García-Raffi. Stealth acoustic materials. Phys. Rev. Applied, 11:054076, May 2019.

[26] B. Song and J. S. Bolton. A transfer-matrix approach for estimating the characteristic impedance and wave numbers of limp and rigid porous materials. The Journal of the Acoustical Society of America, 107:1131-52, 042000.

[27] W. P. Su, J. R. Schrieffer, and A. J. Heeger. Solitons in polyacetylene. Phys. Rev. Lett., 42:16981701, Jun 1979.

[28] G. Theocharis, O. Richoux, V. Romero-García, A. Merkel, and V. Tournat. Limits of slow sound propagation and transparency in lossy, locally resonant periodic structures. New J. Phys., 16(9):093017, 2014.

[29] S. Torquato. Hyperuniformity and its generalizations. Phys. Rev. E, 94:022122, Aug 2016.

[30] S. Torquato, G. Zhang, and F. H. Stillinger. Ensemble theory for stealthy hyperuniform disordered ground states. Phys. Rev. X, 5:021020, May 2015.

[31] M. Xiao, G. Ma, Z. Yang, P. Sheng, Z. Zhang, and C. T. Chan. Geometric phase and band inversion in periodic acoustic systems. Nature Physics, 11(3):240-244, 2015.

[32] M. Xiao, Z. Q. Zhang, and C. T. Chan. Surface impedance and bulk band geometric phases in one-dimensional systems. Phys. Rev. X, 4:021017, Apr 2014.

[33] B. Xie, H. Liu, H. Cheng, Z. Liu, S. Chen, and J. Tian. Acoustic topological transport and refraction in a kekulé lattice. Phys. Rev. Applied, 11:044086, Apr 2019. 